\title{
LISTA DE PARECERISTAS AD HOC DE 2016
}

o final de mais um ano, os Editores Associados da revista Educação \& $A$ Sociedade e a Diretoria do Centro de Estudos Educação e Sociedade 11 (Cedes) agradecem a colaboração de todos os membros de seus conselhos editoriais e colaboradores permanentes e, particularmente, aos pareceristas ad hoc elencados a seguir, que emitiram pareceres e promoveram, com sua experiência e profissionalismo, a arbitragem dos textos submetidos para avaliação neste periódico, durante o ano de 2016.

\section{Adilson Dalben}

Faculdade SESI-SP/Centro Universitário

Salesiano de São Paulo (UNISAL)

Adriana Bauer

Fundação Carlos Chagas (FCC)

\section{Adriana Hoffmann}

Universidade Católica de Petrópolis (UCP)

\section{Adriana Dragone Silveira}

Universidade Federal do Paraná (UFPR)

\section{Alda Junqueira Marin}

Pontifícia Universidade Católica de São Paulo (PUC/SP)

\section{Alejandra Taborda}

Universidad de Córdoba (Colômbia)

Alessandra Arce

Universidade Federal de São Carlos

(UFSCar)

\section{Alex Sander da Silva}

Universidade de Sáo Paulo (USP)

\section{Alexandre Filordi de Carvalho}

Centro Federal de Educação Tecnológica

"Celso Suckow da Fonseca" (CEFET/RJ)

\section{Alexandre Simão Freitas}

Universidade Federal de Pernambuco (UFPE)

\section{Alice Miriam Happ Botler}

Universidade Federal de Pernambuco (UFPE)

\author{
Aline Reali \\ Universidade Federal de São Carlos \\ (UFSCar)
}

\section{Alvaro Moreira Hypolito}

Universidade Federal de Pelotas (UFPel)

\section{Amarílio Ferreira Junior \\ Universidade Federal de São Carlos \\ (UFSCar)}

\section{Ana Cláudia Balieiro Lodi}

Universidade de São Paulo (USP)

\section{Ana Karina Brenner}

Universidade do Estado do Rio de Janeiro (UERJ)

\section{Ana Lúcia Nogueira}

Universidade Estadual de Campinas

(Unicamp)

\author{
Ana Maria Almeida \\ Universidade Estadual de Campinas \\ (Unicamp)
}

\section{Ana Mercês Bahia Bock \\ Pontifícia Universidade Católica de São Paulo (PUC/SP)}

\section{Ana Luiza Bustamante Smolka \\ Universidade Estadual de Campinas (Unicamp)}




\section{Ana Paula Corti}

Instituto Federal de Educação, Ciência e Tecnologia de São Paulo (IFSP)

Anderson Ferrari

Universidade Federal de Juiz de Fora (UFJF)

\section{Anderson RicardoTrevisan}

Centro Universitário Hermínio Ometto (Uniararas)

\section{André Luiz Paulilo}

Universidade Estadual de Campinas

(Unicamp)

\section{Andreu Termes}

Universidade Aberta do Brasil (UAB)

Anete Abramowicz

Universidade Federal de São Carlos

(UFSCar)

\section{Ângela Maria Martins}

Fundação Carlos Chagas (FCC)

Angela R. Maciel De Barros Tamberlini Universidade Federal Fluminense (UFF)

\section{Angelo Cenci}

Universidade de Passo Fundo (UPF)

\section{Bruno Bontempi Júnior}

Universidade de São Paulo (USP)

\section{Bruno Pucci}

Universidade Metodista de Piracicaba (UNIMEP)

\section{Carla Carrochano}

Universidade Federal de São Carlos (UFSCar)

\section{Carlos Artexes Simóes}

Centro Federal de Educação Tecnológica

"Celso Suckow da Fonseca" (CEFET/RJ)

\section{Carolina de Roig Catini}

Universidade Estadual de Campinas (Unicamp)

Cássia Geciauskas Sofiato

Universidade de São Paulo (USP)

\section{Cezar Migliorin}

Universidade Federal Fluminense (UFF)

\section{Claudia Davies}

Fundação Carlos Chagas SP (FCC)

Cláudio Marques Martins Nogueira

Universidade Federal de Minas Gerais (UFMG)

\section{Cristiane Parente de Sá Barreto}

Universidade do Minho (UMinho - Bolsista)

\section{Daniel Mill}

Universidade Federal de São Carlos (UFSCar)

\section{Daniel Soczek}

Centro Universitário Internacional (UNINTER)

\section{Daniele Nunes Henrique Silva}

Universidade de Brasília (UnB)

\section{Décio Gatti}

Universidade Federal de Uberlândia (UFU)

\section{Denise Meyrelles de Jesus}

Universidade Federal do Espírito Santo (UFES)

\section{Dilmeire Sant'anna Ramos Vosgerau} Pontifícia Universidade Católica do Paraná (PUC/PR)

\section{Divino José da Silva}

Universidade Estadual Paulista "Júlio de Mesquita Filho" (UNESP)

\section{Eduardo Calil Oliveira}

Universidade Federal de Alagoas (UFAL)

\section{Elaine Prodócimo}

Universidade Estadual de Campinas

(Unicamp)

\section{Elenice Maria Cammarosano Onofre}

Universidade Federal de São Carlos (UFSCar) 


\section{Eliza Bartolozzi Ferreira}

Universidade Federal do Espírito Santo (UFES)

\section{Elizabeth Macedo}

Universidade do Estado do Rio de Janeiro (UERJ)

\section{Eloísa Dias Gonçalves}

Sociedade Educacional de Santa Catarina (UNISOCIESC)

\section{Eloisa Maia Vidal}

Universidade de São Paulo (USP)

\section{Enicéia Gonçalves Mendes}

Universidade Federal de São Carlos

(UFSCar)

\section{Fabiana de Cássia Rodrigues}

Universidade Estadual de Campinas (Unicamp)

\section{Giovanna Modé Magalhães}

Universidade de São Paulo (USP)

\section{Gisele Masson}

Universidade Estadual de Ponta Grossa (UEPG)

\section{Graça Caldas}

Universidade Estadual de Campinas

(Unicamp)

\section{Gustavo Ferreira Costa Lima}

Universidade Federal da Paraíba (UFPB)

\section{Izabel Augusta Pires Loreto}

Universidade Federal do Rio Grande do Norte (UFRN)

\section{Janete Maria Lins De Azevedo}

Universidade Federal de Pernambuco (UFPE)

\section{Jefferson Mainardes}

Universidade Estadual de Ponta Grossa (UEPG)

\section{João Carlos Simóes}

Centro Federal de Educação Tecnológica

Celso Suckow da Fonseca (CEFET/RJ)

\section{Joáo Ignacio Pires Lucas}

Universidade de Caxias do Sul (UCS)

\section{Joáo Reis Silva Júnior}

Universidade Federal de São Carlos

(UFSCar)

\section{Joáo Wanderley Geraldi}

Universidade Estadual de Campinas

(Unicamp)

\section{José Francisco Soares}

Universidade Federal de Minas Gerais (UFMG)

\section{Jose Manuel Resende \\ Universidade de Lisboa (ULisboa)}

\section{José Rothen}

Universidade Federal de São Carlos

(UFSCar)

\section{Juan José Leiva Olivencia}

Universidad de Málaga (UMA)

\section{Kimi Tomizaki}

Universidade de São Paulo (USP)

\section{Lavínia Magiolino}

Universidade Estadual de Campinas (Unicamp)

\section{Leda Scheibe}

Universidade Federal de Santa Catarina (UFSC)

\section{Lorene dos Santos}

Pontifícia Universidade Católica de Minas Gerais (PUC Minas)

\section{Lucia Pintor Santiso Villas Bôas}

Fundação Carlos Chagas (FCC)

\section{Luciana Coutinho}

Universidade Federal de São Carlos (UFSCar) 


\section{Luciana Rosa Marques \\ Universidade Federal de Pernambuco (UFPE) \\ Luis Armando Gandin \\ Universidade Federal do Rio Grande do \\ Sul (UFRGS) \\ Luís Miguel Carvalho \\ Universidade de Lisboa (ULISBOA) \\ Luísa Álvares Pereira \\ Universidade de Aveiro (UA) \\ Luiz Bezerra Neto \\ Universidade Federal de São Carlos \\ (UFSCar)}

\section{Luiz Roberto Gomes}

Universidade Federal de São Carlos (UFSCar)

\section{Magali Aparecida Silvestre}

Universidade Federal de São Paulo (UNIFESP)

\section{Manuel Sarmento}

Universidade do Minho (UMinho)

\section{Marcelo Burgos}

Pontifícia Universidade Católica do Rio de Janeiro (PUC-Rio)

\section{Marcos Cassin}

Universidade de São Paulo (USP)

\section{Margarita Rosa Sgró}

Universidad Nacional del Centro de la

Provincia de Buenos Aires (UNCPBA)

Maria Aparecida Affonso Moysés

Universidade Estadual de Campinas

(Unicamp)

\section{Maria Beatriz Luce}

Universidade Federal do Rio Grande do Sul (UFRGS)

\section{Maria Benedita Portugal e Mello}

Universidade de Lisboa (ULISBOA)

\section{Maria Cecília Rafael Góes}

Universidade Metodista de Piracicaba (UNIMEP)
Maria Cecília Luiz

Universidade Federal de São Carlos (UFSCar)

\section{Maria Cristina Bezerra}

Universidade Federal de São Carlos (UFSCar)

\section{Maria Clara Di Pierro}

Universidade de São Paulo (USP)

Maria de Lourdes Zanolli

Universidade Estadual de Campinas (Unicamp)

Maria do Rosário Longo Mortatti Universidade Estadual Paulista "Júlio de Mesquita Filho" (UNESP)

Maria Elisa Martins Ladeira

Universidade de São Paulo (USP)

Maria Eugénia Ferráo

Universidade da Beira Interior (UBI)

Maria Glória Gohn

Universidade Estadual de Campinas

(Unicamp)

\section{Maria Graça Jacintho Setton}

Universidade de São Paulo (USP)

\section{Maria Vieira Silva}

Universidade Federal de Uberlândia (UFU)

\section{Marília Pontes Sposito}

Universidade de São Paulo (USP)

\section{Mario Luiz Neves de Azevedo}

Universidade Estadual de Maringá (UEM)

\section{Marisa Bittar \\ Universidade Federal de São Carlos (UFSCar) \\ Marise Nogueira Ramos \\ Universidade do Estado do Rio de Janeiro (UERJ)}


Marta Maria Chagas Carvalho

Universidade de Sorocaba (UNISO)

Mauricio Ernica

Universidade Estadual de Campinas

(Unicamp)

Mônica Correia Baptista

Universidade Federal de Minas Gerais (UFMG)

\section{Mônica Castagna Molina}

Universidade de Brasília (UnB)

\section{Monica Ribeiro Silva}

Universidade Federal do Paraná (UFPR)

\section{Naira Franzoi}

Universidade Federal do Rio Grande do Sul (UFRGS)

\section{Ocimar Alavarse}

Universidade de Sáo Paulo (USP)

Óscar Arias Rojas

Universidad de Chile

Otaviano Augusto Marcondes Helene

Universidade de São Paulo (USP)

\section{Pedro Roberto Jacobi}

Universidade de São Paulo (USP)

Reinaldo Tadeu Boscolo Pacheco

Universidade de São Paulo (USP)

\section{Roberto da Silva}

Universidade de São Paulo (USP)

\section{Rodrigo Cornejo}

Universidad de Chile

Ronaldo Marcos de Lima Araújo

Universidade Federal do Pará (UFPA)

\section{Rosalia Duarte}

Pontifícia Universidade Católica do Rio de Janeiro (PUC-Rio)
Sandra Maria Zákia Lian Sousa

Universidade de Sáo Paulo (USP)

\section{Sandra Regina Garcia}

Universidade Estadual de Londrina (UEL)

\section{Selma Venco}

Universidade Estadual de Campinas (Unicamp)

\section{Sérgio Stoco}

Universidade Federal do ABC Santo André (UFABC)

\section{Sheila Daniela Medeiros dos Santos}

Universidade Federal de Goiás (UFG)

\section{Silvia Helena Vieira Cruz}

Universidade Federal do Ceará (UFC)

\section{Sonia Rummert}

Universidade Federal Fluminense (UFF)

\section{Thiago Alves}

Universidade Federal do Paraná (UFPR)

\section{Timothy Denis Ireland}

Universidade Federal da Paraíba (UFPB)

\section{Valter Roberto Silvério}

Universidade Federal de São Carlos (UFSCar)

\section{Vanda Ribeiro}

Universidade Cidade de São Paulo

(Unicid)

\section{Vanessa Regina de Oliveira Martins}

Universidade Federal de São Carlos

(UFSCar)

\section{Vera Lúcia Jacob Chaves}

Universidade Federal do Pará (UFPA)

Vinício Macedo Santos

Universidade de São Paulo (USP) 